Article

\title{
Modal Analysis of Selected Measuring Bases and Their Impact on the Recorded Level of Surface Accelerations
}

\author{
Krzysztof Robert Czech \\ Department of Geotechnics and Structural Mechanics, Bialystok University of Technology, \\ 15-351 Bialystok, Poland; k.czech@pb.edu.pl
}

Received: 9 April 2020; Accepted: 28 April 2020; Published: 30 April 2020

check for updates

\begin{abstract}
In predicting the impact of vibrations propagated in a ground on newly designed construction objects, it is extremely important to reliably measure the time histories of velocity or acceleration at the site of the planned investment. As some studies show, the method of coupling accelerometers and geophones to the ground can affect not only the level of peak particle accelerations (PPA) or peak particle velocities (PPV) — commonly used in this type of evaluation-but also vibration frequency distribution of recorded signals. This makes it difficult to compare and analyze the results obtained by various research teams. Conclusions based on this type of comparison may be wrong. For this reason, it is extremely important that reliable dynamic measurements related to the propagation of ground surface vibrations should be carried out using not only appropriately selected measuring equipment (with the required sensitivity and measurement ranges-both in the time domain and frequency domain), but also the measurement bases/setups used for mounting various types of transducers, whose natural frequencies will be outside the frequency range relevant to the possible impact of vibrations on buildings and human beings inside. The paper presents the results of modal analyses carried out with the use of Ansys Engineering Simulation and 3D Design Software (based on the Finite Element Method) for three different types of measuring bases used to coupling accelerometers to the ground. Measuring bases selected for numerical analyses were in the form of a stiff steel spike of an X-shaped cross section (a measuring base No. 1) and two steel spikes of L-shaped cross-sections (a measuring bases No. 2 and No. 3). In the places of screwed accelerometers (three different types of IEPE/ICP transducers of varying sensitivity and a weight) point masses were attached to the measuring bases. As a result of the analyses, it was possible to determine the impact of individual methods of coupling of accelerometers to the ground on the reliability of recorded ground surface accelerations. Among others it was concluded that in each analyzed case the lowest natural frequencies of the measuring bases with attached accelerometers significantly exceeded $100 \mathrm{~Hz}$. The widest frequency band free of resonance vibrations can be provided by the measuring base No. 3 $(\mathrm{L} 50 \times 50 \times 5)$.
\end{abstract}

Keywords: modal analysis; measuring bases/setups; coupling to the ground; surface accelerations

\section{Introduction}

An inseparable element of the civilization progress accompanying the dynamic development of industry and trade is the need to erect new industrial facilities, residential and public utility buildings as well as to expand the communication infrastructure enabling effective transport of both people and goods. Unfortunately, this process, apart from positive aspects (such as the increase in the availability of various types of goods and services, communication improvements in the form of new or modernized communication routes, and the appearance of new and faster means of transport) is also associated 
with a negative impact on the environment. It manifests itself not only in the form of pollution and contamination of air, soil, and surface and groundwater; increasingly, in engineering practice we deal with environmental pollution in the form of noise and vibrations. Noise decreases not only the comfort of living, but also the comfort of work-thus negatively affecting its efficiency. In turn, intense vibrations generated by blasting (in mines, quarries, and construction sites $[1,2]$ ), ground vibrations caused by civil engineering works (passing heavy equipment [3,4], soil compaction [5-9], sheet pile driving and pulling out [10,11], pile driving [1,4,5,12], or traffic-induced vibrations [13-16]) can propagate over considerable distances and negatively affect not only the deterioration of living comfort and use, but also the technical condition of nearby located structures [1,4].

In most civilized countries, if a negative impact of vibrations transmitted through the ground on buildings and their users is found or possible to occur, appropriate guidelines and normative regulations apply, which require the investor to commission dynamic measurements, evaluate the impact of existing or predicted vibrations on the planned investment, and if necessary, taking appropriate measures to minimize the negative effects of the planned investment on the environment. These may be measures taken at the vibration source, along the transmission path, or taken at the vibration receiver itself [17] —and if this is not possible activities aimed at modifying the operating parameters of the vibration source (reduction of power/voltage/pressure supplied to the machine or device, change of rotational speed; in the case of traffic vibrations-introduction of restrictions connected with the maximum total weight and vehicle travel speed [18]).

In engineering practice, we do not always deal with an existing source of vibrations and an erected building object that is a receiver of vibrations. Due to the constantly decreasing availability of free areas for development and the increasing density of urban areas, we are now much more likely than in previous years to predict the impact of vibrations on newly designed buildings or predict the impact of new sources of vibrations (e.g., new communication routes) on existing buildings and their users.

Virtually every civilized country has its own regulations on this subject. In the European Union, the key normative acts in the field of measuring vibrations on the ground and the predicted assessment of the impact of vibration on buildings are DIN 4150-3:1999 [19], DIN 45669-2:2005-06 [20], BS 7385-1:1990 [21], BS 7385-2:1993 [22], BS 5228-4:1992 [23], and BS ISO 4866:2010/ISO 4866:2010E [24]. In some countries (such as in New Zealand [4]) it was proposed to adopt identical or like the above normative methods for assessing the impact of vibrations on buildings and people in the building. In other countries (among others in Poland (PN-B-02170:2016-12 [25]) slightly different criteria were applied. The problem is that there are no common, harmonized, and binding (even in the EU) rules for dynamic measurements and acquisition of necessary data related to the vibrations transmitted through the ground and for predicting their impact on buildings and their occupants. In most national standards, the method of coupling accelerometers or geophones to the ground is treated rather perfunctorily or is not specified at all. One of the exceptions is DIN 45669-2:2005-06 [20], which more precisely, in comparison with other standard guidelines indicates possible ways of carrying out this type of measurements using transducers:

- $\quad$ Mounted on spikes (method 1);

- placed on a plate, which is laid by slurrying on a foundation of sand on the ground surface (method 2);

- $\quad$ buried in the ground (method 3); or

- using borehole transducers (method 4).

The second of the methods (method 2) mentioned in DIN 45669-2:2005-06 [20] for measuring surface vibrations transmitted through the ground seems to be the simplest, but according to the author, in the case of more intense vibrations, the degree of coupling of transducers to the ground is insufficient, because the plate with transducers mounted on it may slipping in a horizontal plane relative to the ground surface and even temporarily have decreased contact with the ground in a vertical direction. It can lead to the recording of disturbed time history of accelerations or velocities. 
The last two methods of carrying out measurements (methods 3-4) may require obtaining the appropriate permit for a vertical borehole or an excavation. In both methods, the measurement is quite problematic and involves a significant risk of damage to the transducers. It can also be problematic to ensure proper orientation of the transducers in a hole or a borehole (in accordance with the adopted measuring directions). The measurements should also take into account the fact that the most of surface vibration energy is propagated in the ground medium mainly in the form of Rayleigh waves, which disappear asymptotically to zero with depth, and at a depth of $1 \mathrm{~m}$ it has already been reduced to a fraction [26].

In the first methods (method 1) not much equipment is required. Usually, it provides better ground coupling than measurements using a plate set up on a properly prepared ground surface covered with a thin layer of sand bedding, it is quick, and as indicated by DIN 45669-2:2005-06 [20] suitable for use in many different locations. On the other hand, DIN 45669-2 [20] indicates that the results can be influenced by: Spike geometry, spike length, the height of the transducer above the ground surface, and the position of transducer in relations to the spike's center of mass. In addition, very stiff spikes in well-compacted soil tend to register only localized motions of the upper soil layer.

The lack of a clearly indicated way of carrying out this type of measurements means that in engineering practice and scientific research very diverse solutions are used—often unique only for a given region of the country, or even a scientific center dealing with the propagation of vibrations in a ground medium. Very often they are a combination of the above solutions.

For example, in measurements of ground vibrations described in the report [3] seismic accelerometers (with voltage sensitivity of about $10 \mathrm{~V} / \mathrm{g}$ ) were fastened with screws to the measuring base in the form of a $100 \mathrm{~mm}$ square metal plate with a $200 \mathrm{~mm}$ long spike at each corner.

In the case of the tests described in the Hiller report [5], a set of three uniaxial geophones (SENSOR SM-6 low frequency geophone A-Coil with a sensitivity of $28 \mathrm{~V} / \mathrm{m} / \mathrm{s}$ and a weight of $81 \mathrm{~g}$ ) and additionally a set of uniaxial accelerometers (MONITRAN MTN1100/75 with a sensitivity of $100 \mathrm{mV} / \mathrm{g}$ and a weight of $120 \mathrm{~g}$ ) was screwed to an aluminum cube and next screwed on to $200 \mathrm{~mm}$ long stainless steel spike previously driven fully into the ground.

The cube for attaching accelerometers (KISTLER 8330A3: sensitivity $1.2 \mathrm{~V} / \mathrm{g}$, weight $28.5 \mathrm{~g}$ ) was also used in [6]. However, in this case, the rigid steel cube with three uniaxial accelerometers mounted on its side surfaces was not anchored in the ground in any way by spikes but was pushed into the soil.

A solution like the above was used in the studies [14]. In this case, uniaxial accelerometers (with sensitivity $1 \mathrm{~V} / \mathrm{g}$ and unspecified weight) were mounted to a magnetic base, and then pushed into a previously dug hole in the ground about $20 \mathrm{~cm}$ deep.

In the research conducted by the authors of [27] a triaxial IEPE accelerometer was mounted on "a down hole tool", which was placed in the drilled borehole. The paper does not comprise detailed information about the accelerometer or the measurement base used in the study, nor information about the depth at which the accelerometer was placed in the borehole.

A different approach to the implementation of ground vibration measurements was used in [28], in which accelerometers (most likely uniaxial with a sensitivity of $1 \mathrm{~V} / \mathrm{g}$ and a weight of $210 \mathrm{~g}$ ) were mounted to steel spikes of varying lengths driven into the ground (made of $5 \mathrm{~mm}$ thick steel angle bars and lengths: $500 \mathrm{~mm}, 750 \mathrm{~mm}, 1000 \mathrm{~mm}$, and $1250 \mathrm{~mm}$ ) or screwed to a metal cube with a triangular metal base placed directly on the ground surface. It was found that in the case of accelerometers attached to a metal cube at lower frequencies higher vibration acceleration values are obtained. According to the authors of the work [28], this may be related to so-called "thermal noise". The issue of the impact of the length of steel spikes on the level of recorded vibrations also requires further research.

Various ways of coupling accelerometers to the ground were also analyzed in the paper [29]. The subject of detailed analyses were the results of vibration acceleration measurements (both in time and frequency domain) carried out using: an accelerometer mounted to an aluminum cube (with dimensions of sides equal to $100 \mathrm{~mm}$ ) and beforehand screwed to a concrete block partially embedded 
in the ground, accelerometer mounted to the aluminum cube and set on a bolt driven into the ground, as well as accelerometer bolted to a steel cross with four pins driven into the ground. In the conclusions, the authors state that "the coupling of accelerators to the ground using crosses with four pins driven into the ground is the correct way". A four-point ground anchored steel measuring base in the shape of cross with a screwed metal cubic for mounting accelerometers is also recommended by the Polish standard PN-B-02170: 2016-12 [25].

Measurement setup, in which high sensitivity seismic accelerometers were mounded on an aluminum cube fixed on a concrete block that was partially buried into the ground, was also utilized by authors of the work [30]. To protect accelerometers (capable of measuring ground-borne vibrations from $0.5 \mu \mathrm{g}$ to $0.5 \mathrm{~g}$ in a frequency range between $0.1 \mathrm{~Hz}$ and $1500 \mathrm{~Hz}$ ) from the effects of climatic conditions (such as wind and temperature), an additional protective box with Styrofoam coating at the sites was used. According to the authors of the work [30], the peak values of vibration accelerations occurring at $150 \mathrm{~Hz}$ and $250 \mathrm{~Hz}$ may result from the amplification of background noise and resonance frequency of the vibration measurement setup used in the research.

The impact of different ways of coupling transducers to the ground on the recorded vibration level was also the subject of research conducted by the author of this article. The results of measurements and analyses of the recorded accelerograms were presented, among others, in [31]. Surface vibration accelerations in this case were recorded using: a cross-measuring bases set on a four bolts driven into the ground with screwed aluminum mounting cube (to which uniaxial accelerometers are mounted), a ring-measuring bases set on a three bolts driven into the ground, a two-ring-measuring bases set on a three bolts driven into the ground, and unusual measuring bases in the form of a screw recessed in the ground with miniature triaxial accelerometers mounted on the top of the screw. Conducted research and comparative analyses allowed to formulate the conclusion that among the four ways of coupling accelerometers to the ground, the most reliable are measurements of surface vibrations carried out with the use of measuring bases rigidly connected to the anchors (ring and cross-shaped measuring bases) and embedded in the ground by hammering.

There are many other scientific publications and research reports in which accelerometers during measurements of vibration were simply set on the ground surface (for example [32]) or which do not provide a detailed information about method of coupling measuring transducers to the ground [1,4,33-35].

The abovementioned publications $[28,29,31]$ indicate significant, even several dozen percent differences in the peak values of vibration accelerations recorded using different methods of coupling transducers to the ground. The spectra of recorded vibrations in the frequency domain also differ significantly $[28,29]$.

Such large discrepancies in the results of conducted research significantly impede not only the comparative analysis of the results presented by various research teams, but also may indicate low reliability or even unsuitability of certain solutions. Too low peak values of recorded vibrations may indicate an insufficient degree of coupling of transducers to the ground. Too high level of vibration may in turn indicate the occurrence of resonance vibrations of the measuring base (anchored in the ground or not) with transducers mounted to it.

The purpose of this work is conducting numerical simulations in Ansys Engineering Simulation Software and identification of modal parameters (in particular natural frequencies) of the selected three types of measuring bases in the form of spikes with attached to them various types of accelerometers, as well as determining reliability of surface vibration accelerations recorded with the use each of them.

\section{Measurement Bases/Setups}

In the paper it has been assumed that three different ways of coupling transducers to the ground belonging to the first group of methods described in DIN 45669-2:2005-06 [20] (i.e., methods based on transducers mounted on spikes) will be subjected to numerical simulations related to the identification of natural frequencies and the corresponding mode shapes of vibrations. 
The first of the ways of coupling accelerometers to the ground analyzed in the work (the measuring base No. 1-in accordance with DIN 45669-2:2005-06 [20] and DIN EN 10056-1 [36]) uses steel spikes hammered into the ground of about $500 \mathrm{~mm}$ long of an X-shaped cross section formed of two angles of about $30 \times 30 \times 4 \mathrm{~mm}$. On one side the spike ends with a pointed end about $150 \mathrm{~mm}$ long. A flat plate of $60 \times 60 \times 10 \mathrm{~mm}$ is attached to the second, upper end of the spike. This solution allows mounting miniature triaxial accelerometer or one uniaxial accelerometer in the vertical direction to the upper surface of a flat bar. Due to the insufficient thickness, the flat bar does not have a sufficiently large contact surface for stable fastening of uniaxial accelerometers in the horizontal plane. For this reason, it was necessary to design a suitable mounting device (so-called adapter or mounting head), which, when attached to the flat bar, would allow measurements of vibration accelerations also in the horizontal plane using not only miniature triaxial accelerometers, but also much more precise, and at the same time heavier and larger seismic accelerometers. The mounting head for attaching accelerometers to the measuring base was designed as a lightweight aluminum cube with a $60 \mathrm{~mm}$ side with four through holes. The mounting head is screwed to the upper surface of the measuring base with Allen screws type DIN-912 M6.

The measuring base No. 2 was adopted in the form of a steel spike with an L-shaped cross-section formed from an angle iron of about $30 \times 30 \times 4 \mathrm{~mm}$ - of identical length $(500 \mathrm{~mm})$ and pointed end (150 mm long) as in the case of measuring base No. 1 (also in accordance with guidelines DIN 45669-2:2005-06 [20] and DIN EN 10056-1 [36]). As before, the cubic mounting head is required to mount a set of uniaxial accelerometers in all three measuring directions.

The measuring base No. 3 was designed as a steel spike with L-shaped cross-section hammered into the ground (made out of angle iron of about $50 \times 50 \times 5 \mathrm{~mm}$ section and $500 \mathrm{~mm}$ long) with welded flat bar (5 mm thick)—as described and drawings contained in [28] as well as in UNI 9916:2004 [37] and UNI 5783-66 [38] standards. Location of the bottom of the flat bar was assumed at a height of $445 \mathrm{~mm}$ from the pointed end of the base (it is also the depth of base anchoring). Threaded holes (1/4-28 UNI) for direct fixing of accelerometers have been made in the flat bar and on the side surfaces of the angle slightly above the flat bar (in this solution it is not necessary to use any additional devices for mounting the accelerometers). L-shaped bar of $40 \mathrm{~mm}$ length was sharp-pint ended (detailed dimensions of the measurement base were adopted based on descriptions and the proportions of the drawings contained in the work [28]).

Geometric models of the measurement bases analyzed in the work are shown in Figure 1.

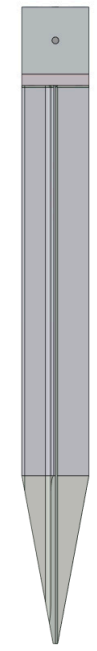

(a)

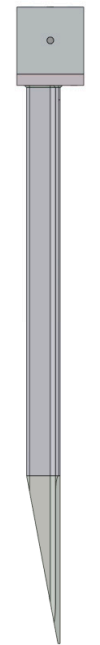

(b)

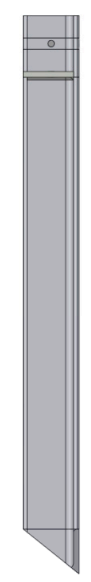

(c)

Figure 1. The measuring bases: (a) Spike with $X$-shaped cross section $(2 \times 30 \times 30 \times 4 \mathrm{~mm})$ with attached mounting head, (b) spike with L-shaped cross section $(30 \times 30 \times 4 \mathrm{~mm})$ with mounting head, (c) L-shaped section spike $(50 \times 50 \times 5 \mathrm{~mm})$. 


\section{Numerical Modelling}

Three-dimensional geometric models of measuring bases were prepared in Ansys Space Claim software (19.2, Ansys, Inc., Canonsburg, PA, USA, 2019). Numerical analyses aimed at determining the modal base parameters were carried out using Ansys Engineering Simulation and 3D Design Software (19.2, Ansys, Inc., 2019) (based on the Finite Element Method-FEM). Verification of numerical analyses was carried out on the latest version of Ansys software (2020 R1, Ansys, Inc., 2020).

For the needs of numerical analyses, all steel elements (sections: $50 \times 50 \times 5 \mathrm{~mm}, 30 \times 30 \times 4 \mathrm{~mm}$, $5 \mathrm{~mm}$ and $10 \mathrm{~mm}$ thick flat bars welded to them, bolts, welds were matched with typical parameters adopted for steel (a Young's modulus $E=210 \mathrm{GPa}$, a Poisson's ratio v $=0.3$, a density $\rho=7850 \mathrm{~kg} / \mathrm{m}^{3}$ ). Additional mounting devices necessary to screw the accelerometers to the measuring bases were attributed with material parameters as for "Aluminum 2024-T4, 2024-T351" $(\mathrm{E}=73.1 \mathrm{GPa}, \mathrm{v}=0.33$, $\rho=2780 \mathrm{~kg} / \mathrm{m}^{3}$ ).

During the three-dimensional modelling of individual measuring bases, efforts were made to reproduce their construction and real work as accurately as possible. Therefore, welds between the angle bars and angle bars and flat bars welded to them were additionally modelled.

The discretization of individual geometric models was carried out using solid finite elements type Hex20 and in places where this was not possible solid elements type Tet10 were applied. After initial discretization of individual models of the measuring bases, the mesh of nodes was densified until it was found that further increasing of the mesh density practically did not affect the change in the value of the results obtained.

The effects of discretization of individual models in the form of a summary including the final number of nodes, the number of finite elements and the maximum size of the finite element are given in Table 1.

Table 1. Division into nodes and finite elements of the measurement bases.

\begin{tabular}{cccc}
\hline & Measuring Base No. 1 & Measuring Base No. 2 & Measuring Base No. 3 \\
\hline Nodes & $358,834(222,670)$ & $209,982(111,552)$ & 193,124 \\
Elements & $164,467(74,512)$ & $103,594(35,509)$ & 48,137 \\
Max body sizing & $3 \mathrm{~mm}(2 \mathrm{~mm})$ & $3 \mathrm{~mm}(2 \mathrm{~mm})$ & $2 \mathrm{~mm}$ \\
\hline \multicolumn{2}{c}{ The number of nodes and finite elements without mounting head are given in brackets. }
\end{tabular}

Contacts of individual elements of the measuring bases were modelled in most cases as "bounded". The exception are the contact surfaces between the screws and the mounting head as well as the mounting head and flat bar, in which "no separation" linear contacts were used. Unfortunately, due to the planned type of analysis (modal analysis), it was not possible to use non-linear "frictional" or "frictionless" contacts, because all these contacts will be reduced to their linear counterparts anyway.

In order to obtain reliable results, numerical simulations in Ansys Engineering Software (19.2, Ansys, Inc., Canonsburg, PA, USA, 2019) were carried out for measuring bases with attached point masses in the places of screwed accelerometers. In the case of the measurement bases No. 1 to No. 3, analyses were carried out for three types of IEPE/ICP transducers: Brüel \& Kjær 8340-type (Nærum, Denmark) uniaxial seismic accelerometer with a sensitivity of $10 \mathrm{~V} / \mathrm{g}$ and a weight of $0.775 \mathrm{~kg}$ (identical in terms of measuring sensitivity and weight as uniaxial accelerometers model 3191A by Dytran Instruments, Inc., Chatsworth, CA, USA), low frequency uniaxial accelerometers model 3187D from Dytran Instruments, Inc. with sensitivity $0.5 \mathrm{~V} / \mathrm{g}$ and weight $0.122 \mathrm{~kg}$, as well as miniature triaxial accelerometers model 356B18 by PCB Piezotronics, Inc. (Depew, NY, USA) with sensitivity $1 \mathrm{~V} / \mathrm{g}$ and a weight with a magnetic base of $0.041 \mathrm{~kg}$.

The final discretization of the individual measurement bases is shown in Figure 2 (in the case of measuring bases No. 1 and No. 2 with mounted lightweight aluminum heads for mounting accelerometers). 


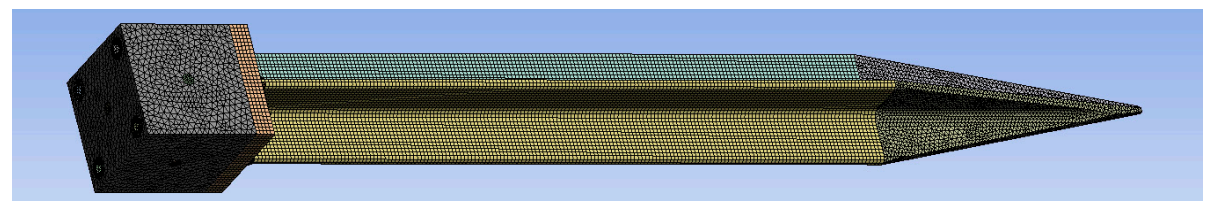

(a)

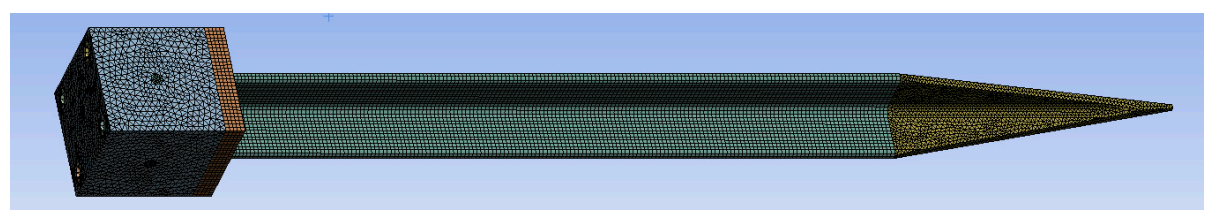

(b)

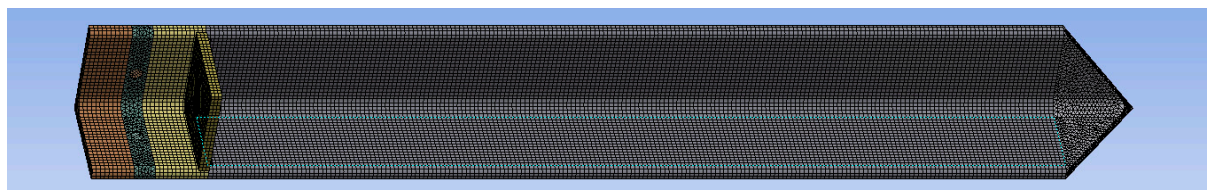

(c)

Figure 2. The measuring bases after meshing: (a) No. 1, (b) No. 2, (c) No. 3.

In the case of modal analysis conducted with the use of engineering software based on the Finite Element Method algorithm, it is possible to determine modal parameters of structure without defining any boundary conditions. However, the mode shapes obtained in this way (so-called free modes of structure) will be far from reality because they will in no way consider the interaction between the ground and the measurement bases embedded in the ground.

Another possible solution is to model the structure on an elastic foundation or blocking selected degrees of freedom of construction (for example, in the case of piles, a common method is a pile fixed at the head [39]). In the case of modal analyses of small-scale bases for measuring vibration in the ground, this solution, unfortunately, cannot be used. For this reason, in the planned numerical analyses, it was initially decided on a simplified solution in the form of simulating interaction at the interface of the ground and the element embedded in it by applying boundary conditions in the form of "flexible support", wherever there is contact between the measuring base and the ground.

Given the very small cross-section of the spikes of the measurement bases embedded in the ground (significantly smaller not only than piles, but also smaller than micro piles), the elastic support values from a much wider range (from $0.005 \mathrm{~N} / \mathrm{mm}^{3}$ to $0.1 \mathrm{~N} / \mathrm{mm}^{3}$ ) were adopted for numerical analyses than it would result from design practice in the case of modelling flexible foundation on the basis of various types of buildings.

The analysis of preliminary results showed that in the case of the lowest eigenmodes frequencies (with frequencies in the range from $0 \mathrm{~Hz}$ to several dozen $\mathrm{Hz}$ ) rigid body motion occurs. Further mode shapes, however, could not be considered reliable.

Unfortunately, it is not possible to perform a reliable modal analysis for three-dimensional measurement bases anchored in a soil medium, considering the non-linear interaction that occurs at the interface between the measurement base and the ground. In modal analysis it is assumed that the structure being the subject of analysis or experimental research is linear. The soil medium is not linear. Even if the soil medium is modelled as homogeneous and isotropic, and the contact of the ground with the measurement base is assumed to be of the "bounded" or "no separation" type, the mass of the soil itself will cause the identified eigenmodes to be largely vibrations of the ground body. Therefore, in the next stage of numerical analyses, a compromise solution was decided, in which the measurement bases were modelled in a cylindrical soil block with real rigidity and mass selected so that it did not exceed $1 \%$ of the mass of the measuring bases with the heaviest seismic transducers mounted. 
Considering the computational capabilities of modern efficient workstations and the real small range of interaction occurring between the measurement bases, a soil body with a diameter of $200 \mathrm{~cm}$ and a height of $100 \mathrm{~cm}$ was adopted for numerical simulations. The adopted dimensions of the soil body correspond to the conditions that we can provide in laboratory conditions for the needs of any possible experimental or operational modal analyses.

Soil parameters for numerical simulations were adopted from geotechnical tests carried out at the Logistics Center Lososna in North-Eastern Poland [40] (homogeneous loose and medium dense coarse sands (cSa) with a density $1844 \mathrm{~kg} / \mathrm{m}^{3}(\rho)$, a Young's modulus $\mathrm{E}_{0}$ in the range of 90.1-116.2 MPa and a Poisson's ratio $\mathrm{v}=0.264-0.309$ ). Eventually, the following, averaged mechanical soil parameters were adopted for numerical analyses: $\mathrm{E}_{0}=100 \mathrm{MPa}$ and $\mathrm{v}=0.3$. In order to check what is the impact of a change in Young's modulus of the soil medium on the frequency distribution of the natural vibrations of the measurement bases, it was additionally assumed that modal analyses will also be carried out for soils with a modulus equal to $75 \mathrm{MPa}$ and $125 \mathrm{MPa}$.

Assuming the volume density of the soil of $0.01 \mathrm{~kg} / \mathrm{m}^{3}$, the total weight of the soil within the adopted body does not exceed $0.032 \mathrm{~kg}$ and constitutes a maximum of $0.7 \%$ of the heaviest measuring base (No. 1) with mounted seismic accelerometers. A soil body modelled this way, with fixed displacement of the bottom surface in the vertical direction $(Z)$ and fixed degrees of freedom in a perpendicular and parallel directions to the side surface ( $X$ and $Y$ degrees of freedom in cylindrical coordinate system) has no tendency to natural vibrations in the frequency range up to at least several $\mathrm{kHz}$ 's - so it should not disturb the natural frequency vibrations of much stiffer and heavier measurement bases anchored in it. Preliminary modal analyses of the measuring bases embedded in such a modelled soil body showed that further reduction of the soil volume density practically does not affect the identified natural frequencies (in the case of measuring base No. 1 with seismic accelerometers mounted, maximum differences of $0.1 \mathrm{~Hz}$ when the soil density changes from $0.01 \mathrm{~kg} / \mathrm{m}^{3}$ to $0.001 \mathrm{~kg} / \mathrm{m}^{3}$ ). A similarly negligible effect on the obtained natural frequencies of the measurement bases has further densification of the mesh of nodes of soil body or reduction of its diameter.

The soil body adopted for analysis after the discretization process (with a significantly concentrated mesh of nodes in the area of contact of the measuring base with the ground) is shown in Figure 3 (about 1.9-2.2 million nodes with the measurement base).

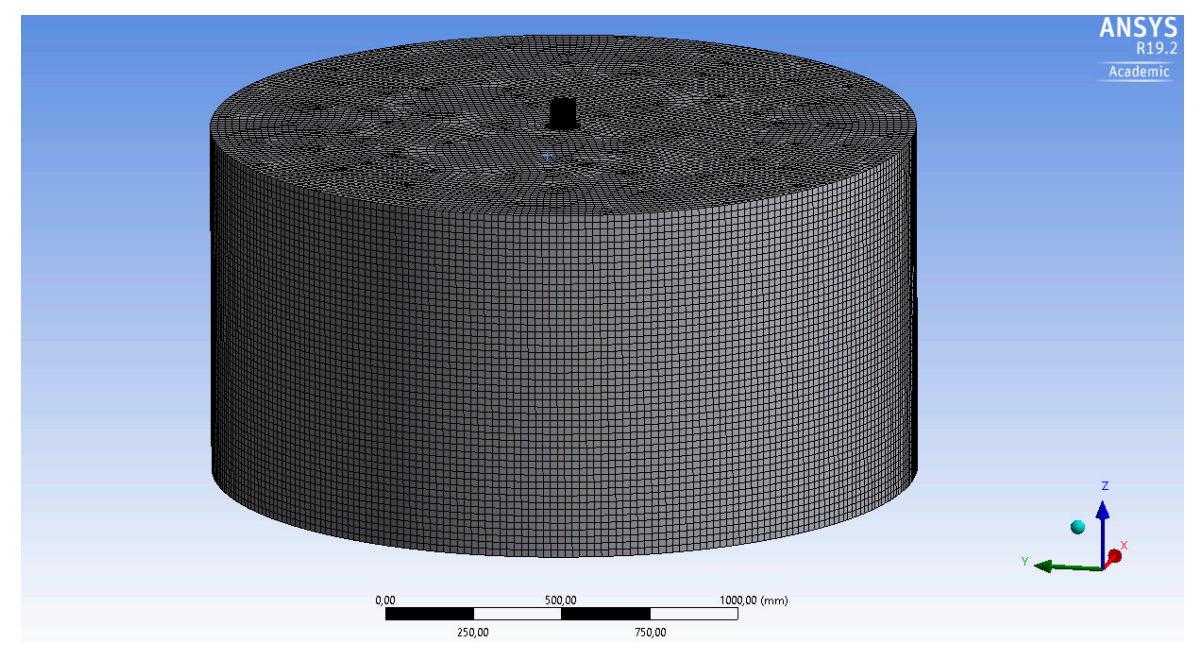

Figure 3. A discrete model of a soil body with embedded measuring base.

Modal analyses can be carried out with or without damping. Damping should necessarily be considered for highly damped structures. However, in most modal analyses, this factor is ignored. Considering the simplifications already made in the modelling of the soil body and the low values of damping of the material from which the measurement bases were made (steel and aluminum) in 
the planned modal analyses, it was assumed that they will be implemented without taking damping into account.

\section{Results of Analyses}

Exemplary eigenmodes (the first modes) of individual measuring bases are shown in Figure 4.

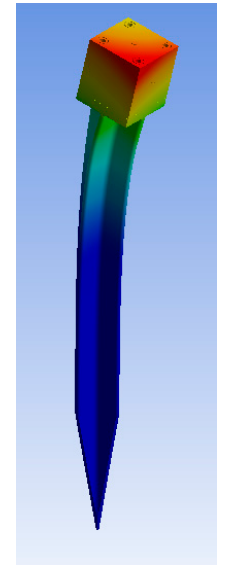

(a)

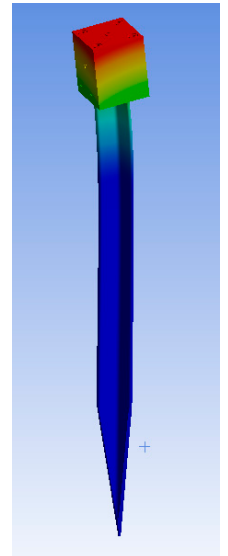

(b)

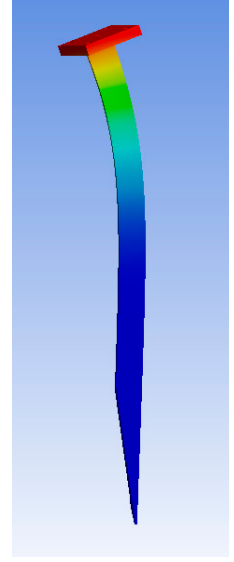

(c)

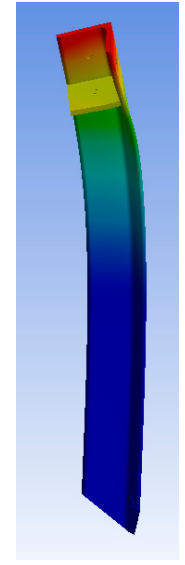

(d)

Figure 4. The lowest mode shapes of the measurement bases: (a) No. 1, (b) No. 2, (c) No. 2-without aluminum mounting head, (d) No. 3.

Figure 5 shows the vibration of the measuring bases inside a soil body.

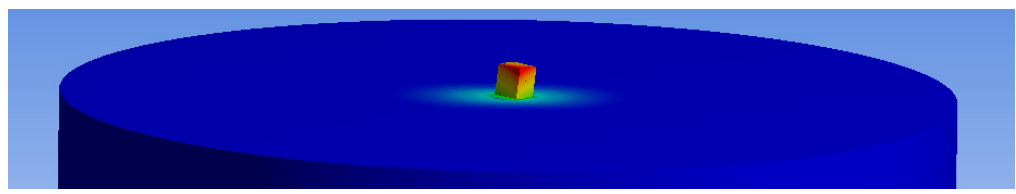

(a)

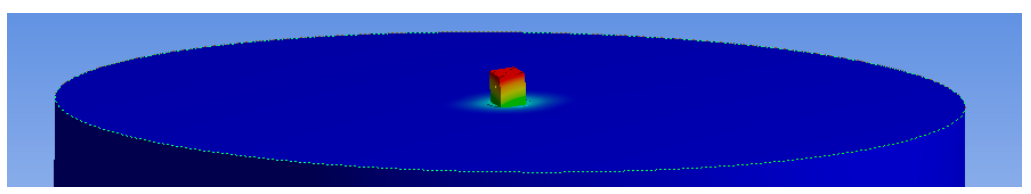

(b)

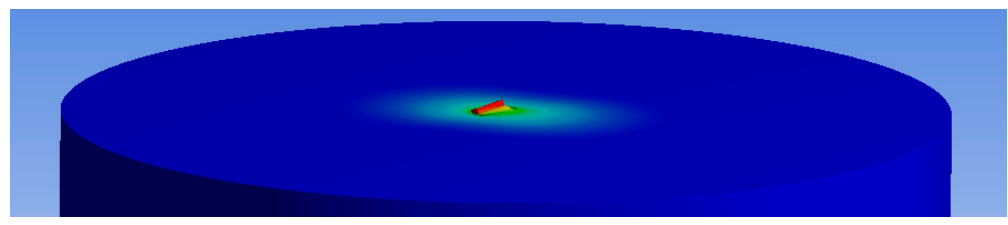

(c)

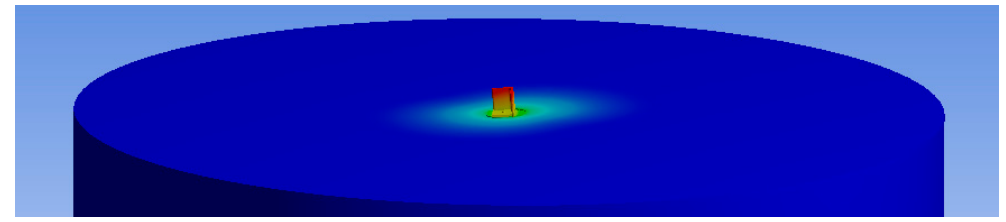

(d)

Figure 5. The first lowest eigenmodes of the measuring bases inside soil body (a) No. 1, (b) No. 2, (c) No. 2-without aluminum mounting head, (d) No. 3. 
The results of conducted numerical simulations related to the determination of modal parameters are summarized in Tables 2-10. Each table shows three lowest natural frequencies. In the case of measurement bases No. 1 and No. 2 miniature 3D accelerometer (PCB Piezotronics, model 356B18) was mounted via a magnetic base directly to the upper surface of the steel flat bar mounting bases eliminating the redundant aluminum assembly head (Tables 4 and 7).

Table 2. Natural frequencies-the measuring base No. $1(2 \times$ L30 $\times 30 \times 4)$ with a set of 3 uniaxial seismic accelerometers: Brüel \& Kjær type 8340/Dytran Instruments type 3191A.

\begin{tabular}{cccc}
\hline Mode & $\mathbf{E}_{\mathbf{o}}=\mathbf{7 5} \mathbf{M P a}$ & $\mathbf{E}_{\mathbf{o}}=\mathbf{1 0 0} \mathbf{M P a}$ & $\mathbf{E}_{\mathbf{o}}=\mathbf{1 2 5} \mathbf{M P a}$ \\
\hline 1 & 196.5 & 218.0 & 236.3 \\
2 & 211.0 & 234.3 & 253.9 \\
3 & 385.6 & 442.7 & 492.2 \\
\hline
\end{tabular}

Table 3. Natural frequencies-the measuring base No. $1(2 \times$ L30 $\times 30 \times 4)$ with a set of 3 uniaxial seismic accelerometers: Dytran Instruments type 3187D.

\begin{tabular}{cccc}
\hline Mode & $\mathrm{E}_{\mathbf{o}}=\mathbf{7 5} \mathbf{M P a}$ & $\mathrm{E}_{\mathbf{o}}=\mathbf{1 0 0} \mathbf{M P a}$ & $\mathrm{E}_{\mathbf{o}}=\mathbf{1 2 5} \mathbf{M P a}$ \\
\hline 1 & 325.5 & 363.6 & 396.0 \\
2 & 341.0 & 381.3 & 415.4 \\
3 & 507.0 & 583.9 & 651.2 \\
\hline
\end{tabular}

Table 4. Natural frequencies-the measuring base No. $1(2 \times \mathrm{L} 30 \times 30 \times 4)$ with a miniature triaxial accelerometer: PCB Piezotronics type 356B18.

\begin{tabular}{cccc}
\hline Mode & $\mathbf{E}_{\mathbf{o}}=\mathbf{7 5} \mathbf{M P a}$ & $\mathrm{E}_{\mathbf{o}}=\mathbf{1 0 0} \mathbf{M P a}$ & $\mathrm{E}_{\mathbf{o}}=\mathbf{1 2 5} \mathbf{M P a}$ \\
\hline 1 & 627.4 & 718.7 & 798.5 \\
2 & 628.8 & 725.5 & 807.4 \\
3 & 634.0 & 726.7 & 810.5 \\
\hline
\end{tabular}

Table 5. Natural frequencies-the measuring base No. $2(\mathrm{~L} 30 \times 30 \times 4)$ with a set of 3 uniaxial seismic accelerometers: Brüel \& Kjær type 8340/Dytran Instruments type 3191A.

\begin{tabular}{cccc}
\hline Mode & $\mathrm{E}_{\mathbf{o}}=\mathbf{7 5} \mathbf{M P a}$ & $\mathrm{E}_{\mathbf{o}}=\mathbf{1 0 0} \mathbf{M P a}$ & $\mathrm{E}_{\mathbf{o}}=\mathbf{1 2 5} \mathbf{M P a}$ \\
\hline 1 & 150.3 & 167.0 & 181.4 \\
2 & 172.6 & 192.0 & 208.5 \\
3 & 384.7 & 433.2 & 475.4 \\
\hline
\end{tabular}

Table 6. Natural frequencies-the measuring base No. $2(\mathrm{~L} 30 \times 30 \times 4)$ with a set of 3 uniaxial seismic accelerometers: Dytran Instruments type 3187D.

\begin{tabular}{cccc}
\hline Mode & $\mathbf{E}_{\mathbf{o}}=\mathbf{7 5} \mathbf{M P a}$ & $\mathrm{E}_{\mathbf{o}}=\mathbf{1 0 0} \mathbf{M P a}$ & $\mathrm{E}_{\mathbf{o}}=\mathbf{1 2 5} \mathbf{M P a}$ \\
\hline 1 & 256.1 & 285.9 & 311.6 \\
2 & 293.3 & 327.5 & 356.8 \\
3 & 549.8 & 630.7 & 698.0 \\
\hline
\end{tabular}

Table 7. Natural frequencies-the measuring base No. $2(\mathrm{~L} 30 \times 30 \times 4)$ with a miniature triaxial accelerometer: PCB Piezotronics type 356B18.

\begin{tabular}{cccc}
\hline Mode & $\mathbf{E}_{\mathbf{o}}=\mathbf{7 5} \mathbf{M P a}$ & $\mathbf{E}_{\mathbf{o}}=\mathbf{1 0 0} \mathbf{M P a}$ & $\mathbf{E}_{\mathbf{o}}=\mathbf{1 2 5} \mathbf{M P a}$ \\
\hline 1 & 636.6 & 727.5 & 807.2 \\
2 & 658.6 & 751.6 & 832.9 \\
3 & 768.8 & 886.0 & 988.6 \\
\hline
\end{tabular}


Table 8. Natural frequencies-the measuring base No. $3(\mathrm{~L} 50 \times 50 \times 5)$ with a set of 3 uniaxial seismic accelerometers: Brüel \& Kjær type 8340/Dytran Instruments type 3191A.

\begin{tabular}{cccc}
\hline Mode & $\mathbf{E}_{\mathbf{o}}=\mathbf{7 5} \mathbf{M P a}$ & $\mathrm{E}_{\mathbf{o}}=\mathbf{1 0 0} \mathbf{M P a}$ & $\mathbf{E}_{\mathbf{o}}=\mathbf{1 2 5} \mathbf{M P a}$ \\
\hline 1 & 249.0 & 278.5 & 303.6 \\
2 & 268.6 & 302.0 & 330.1 \\
3 & 407.6 & 468.0 & 520.5 \\
\hline
\end{tabular}

Table 9. Natural frequencies - the measuring base No. $3(\mathrm{~L} 50 \times 50 \times 5)$ with a set of 3 uniaxial seismic accelerometers: Dytran Instruments type 3187D.

\begin{tabular}{cccc}
\hline Mode & $\mathbf{E}_{\mathbf{o}}=\mathbf{7 5} \mathbf{M P a}$ & $\mathbf{E}_{\mathbf{o}}=\mathbf{1 0 0} \mathbf{M P a}$ & $\mathbf{E}_{\mathbf{o}}=\mathbf{1 2 5} \mathbf{M P a}$ \\
\hline 1 & 456.8 & 514.9 & 564.5 \\
2 & 478.7 & 543.2 & 598.1 \\
3 & 560.5 & 646.4 & 721.7 \\
\hline
\end{tabular}

Table 10. Natural frequencies-the measuring base No. $3($ L50 $\times 50 \times 5)$ with a miniature triaxial accelerometer: PCB Piezotronics type 356B18.

\begin{tabular}{cccc}
\hline Mode & $\mathbf{E}_{\mathbf{o}}=\mathbf{7 5} \mathbf{M P a}$ & $\mathbf{E}_{\mathbf{o}}=\mathbf{1 0 0} \mathbf{M P a}$ & $\mathbf{E}_{\mathbf{o}}=\mathbf{1 2 5} \mathbf{M P a}$ \\
\hline 1 & 587.4 & 668.6 & 738.5 \\
2 & 597.5 & 683.8 & 758.4 \\
3 & 608.2 & 701.5 & 783.6 \\
\hline
\end{tabular}

\section{Discussion}

In engineering practice, there are present both natural sources of excitation (earthquakes, winds), as well as man-made sources of excitation (traffic, construction, blasting). As cited in BS ISO 4866:2010 [24]: "The frequency range of interest depends upon the spectral content of the excitation and upon the mechanical response of the structure". Therefore, in [24] standard, frequencies from a very wide range: From $0.1 \mathrm{~Hz}$ to $500 \mathrm{~Hz}$ are indicated as important from the point of view of the structure receiving vibrations. In the above standard, however, a record can be found stating that most structural damage from man-made sources occurs in the frequency range from $1 \mathrm{~Hz}$ to $150 \mathrm{~Hz}$.

From the point of view of the possible impact of recorded vibrations on newly designed buildings, we are basically interested in reliable measurement of vibrations propagated in a soil medium with frequencies in a slightly narrower frequency band from $0.5-1.0 \mathrm{~Hz}$ to $100 \mathrm{~Hz}[19,25]$. In this frequency band the measuring bases with transducers mounted on them should not show any natural frequencies.

Analyzing the results of numerical simulations carried out in Ansys Engineering Simulation Software, it should be stated that the weight of accelerometers mounted on them has a dominant impact on the natural frequencies of the measuring bases. The higher the total weight of transducers-the lower the values of the natural frequencies of the measuring bases anchored in the ground, and vice versa, the lower the soil stiffness-the higher the natural frequencies of the measuring bases. For the most accurate and also the heaviest uniaxial seismic accelerometers (type 8340 from Brüel \& Kjær with a sensitivity of $10 \mathrm{~V} / \mathrm{g}$ and a weight of $0.775 \mathrm{~kg}$ ), the lowest natural frequencies of the measuring base depending on the adopted Young's modulus of soil $\left(\mathrm{E}_{0}=75-125 \mathrm{MPa}\right)$ are as follows (Tables 2, 5 and 8):

- $\quad 150-181 \mathrm{~Hz}$-for the measuring base No. $2(\mathrm{~L} 30 \times 30 \times 4 \mathrm{~mm})$;

- $197-236 \mathrm{~Hz}$-for the measuring base No. $1(2 \times \mathrm{L} 30 \times 30 \times 4 \mathrm{~mm})$;

- $\quad$ and 249-304 Hz-for the measuring base No. $3(\mathrm{~L} 50 \times 50 \times 5 \mathrm{~mm})$.

The lowest natural frequencies of the same measuring bases fixed with much lighter single-axis low-frequency accelerometers (Dytran Instruments type 3187D with a sensitivity of $0.5 \mathrm{~V} / \mathrm{g}$ and a weight of $0.122 \mathrm{~kg}$ ) are higher by at least $100 \mathrm{~Hz}$, as listed below (Tables 3, 6 and 9): 
- $\quad 256-312 \mathrm{~Hz}$-for the measuring base No. $2(\mathrm{~L} 30 \times 30 \times 4 \mathrm{~mm})$;

- $\quad 326-396 \mathrm{~Hz}$-for the measuring base No. $1(2 \times \mathrm{L} 30 \times 30 \times 4 \mathrm{~mm})$;

- and $457-564 \mathrm{~Hz}$-for the measuring base No. $3(\mathrm{~L} 50 \times 50 \times 5 \mathrm{~mm})$.

From the point of view of the reliability of vibration measurements conducted with the use of the measurement bases presented in the paper, the best solution seems to be the one in which weight of measuring transducers and additional devices necessary for their installation can be reduced to a minimum. This situation occurs when, instead of a set of single-axis transducers, one miniature triaxial accelerometer is applied (for instance, type 356B18 from PCB Piezotronics Inc. with a sensitivity of $1 \mathrm{~V} / \mathrm{g}$ and a weight with a magnetic base of $0.041 \mathrm{~kg}$ ). An additional head for mounting accelerometers is then unnecessary. All measuring bases analyzed in this article, equipped with a 3-axis accelerometer theoretically enable reliable measurement of surface accelerations with frequencies even above $500 \mathrm{~Hz}$ (Tables 4, 7 and 10). In the case of this type of accelerometers, it should be noted, however, that miniature accelerometers with such a light weight usually have a much higher level of Equivalent Electrical Noise Floor than many times heavier uniaxial seismic accelerometers (weighing ca. $0.775 \mathrm{~kg}$ ) and several times heavier low-frequency accelerometers (weighing over $100 \mathrm{~g}$ and a twice lower sensitivity of $0.5 \mathrm{~V} / \mathrm{g}$ ).

In the case of dynamic measurements carried out with the use of standard uniaxial accelerometers, the measurement base No. 3 seems to be the best as it does not require any additional mounting elements and ensures the widest frequency band free of resonance vibrations (Tables 8-10).

\section{Conclusions}

Reliable measurement of surface vibrations propagated in a soil medium requires the use of such a coupling of measuring transducers to the ground that during the measurement there is no resonance vibration of the measuring bases with mounted to them transducers or other distortions of recorded signals in the range of amplitudes and frequencies. From the point of view of the predicted assessment of the impact of vibrations on buildings structures, we are generally interested in vibration frequencies in the band at least from about $0.5-1.0 \mathrm{~Hz}$ to $100 \mathrm{~Hz}[5,19,22]$. Considering the above, and based on the results of modal analyses carried out using the Ansys Engineering Simulation and 3D Design Software, it should be stated that:

- All three methods of coupling accelerometers to the ground by using so-called "spikes", theoretically provide a reliable measurement of surface vibration in terms of evaluating the predicted effects of recorded vibrations on the building structures (in each analyzed case the lowest natural frequencies of the measurement bases with attached accelerometers significantly exceeded $100 \mathrm{~Hz}$ );

- the weight of accelerometers mounted to measuring bases has the dominant influence on the natural frequencies of these measurement bases (the greater the total mass of transducers mounted-the lower the natural frequencies of the measuring bases embedded in the ground);

- the distribution of the natural frequencies of the measuring bases is affected by soil stiffness to a slightly smaller extent (the greater the soil stiffness modulus-the higher the identified natural frequencies of the measuring bases embedded in the ground);

- the optimal solution is a compromise in which the weight of the devices used to coupling transducers to the ground is minimized while using accelerometers with the highest possible sensitivity; and

- the widest frequency band in which there should be no resonance vibrations of the measuring bases with accelerometers mounted to them can be provided by the measuring base No. $3($ L50 $\times 50 \times 5$ ).

The conclusions presented above are based on a simplified numerical model, which was developed for the needs of a linear modal analysis. In real conditions, the contact between the measuring bases/setups driven into the ground in the form of spikes and the ground is not linear. Also, the soil 
medium is neither homogeneous nor isotropic. Usually, when driving measurement bases in the soil, its surface layers loosen. For this reason, before measuring surface vibrations, the soil around the measuring bases must be well compacted. Unfortunately, under real conditions, much lower dominant natural frequencies of this type of measuring base with mounted transducers should be expected anyway.

Bearing in mind the reliability of measurements of surface acceleration, it is advisable to perform analogous numerical simulations for other solutions commonly used for this purpose (e.g., accelerometers mounded on an aluminum cube fixed on a concrete block and partially buried into the ground, cross-measuring bases set on a four bolts driven into the ground, and plate bases set on a three bolts driven into the ground). For selected types of measurement bases it is planned to conduct experimental or operational modal analysis in laboratory conditions.

Funding: This work: carried out at Bialystok University of Technology, was supported by Polish financial resources on science under project no. WZ/WB-IIL/2/2020.

Acknowledgments: The research was carried out with the use of equipment purchased from EU funds as part of the "INNO-EKO-TECH" project and software made available to the Bialystok University of Technology under a partnership agreement with ANSYS, Inc. and MESco Sp. z o.o. The author gratefully acknowledges Adam Tkaczuk for help in numerical simulations.

Conflicts of Interest: The author declares no conflict of interest. The funders had no role in the design of the study; in the collection, analyses, or interpretation of data; in the writing of the manuscript, or in the decision to publish the results.

\section{References}

1. New, B.M. Ground Vibration Caused by Civil Engineering Works; Ground Engineering Division; Research Report 53; Higways and Structures Department, Transport and Road Research Laboratory: Crowthorne, Berkshire, UK, 1986.

2. Kim, D.K.; Lee, J.S. Propagation and Attenuation Characteristics of Various Ground Vibrations. Soil Dyn. Earthq. Eng. 2000, 19, 115-126. [CrossRef]

3. Martin, D.J. Ground Vibrations Caused by Road Construction Operations; TRRL Supplementary Report 328; Transport and Road Research Laboratory, Department of the Environment, Department of Transport: Crowthorne, Berkshire, UK, 1977.

4. Cenek, P.D.; Sutherland, A.J. Ground Vibration from Road Construction; Research Report 485; New Zealand Transport Agency: Wellington, New Zealand, 2012.

5. Hiller, D.M.; Crabb, G.I. Groundborne Vibration Caused by Mechanised Construction Works; Report 429; Transport Research Laboratory: Crowthorne, Berkshire, UK, 2000.

6. Hao, H. Measurement of Plate Compaction-Induced Ground Vibrations. In Proceedings of the 23rd Australian Conference on the Mechanics of Structures and Materials (ACMSM23), Byron Bay, NSW, Australia, 9-12 December 2014; Southern Cross University: Lismore, Australia, 2014; Volume II, pp. 727-732.

7. Wang, C. Effect of Hydraulic Plate Compactor and Lift Thickness on Utility Trench Backfill Compaction. Master's Thesis, Department of Civil and Environmental Engineering, The Pennsylvania State University, State College, PA, USA, 2015.

8. Czech, K.; Gosk, W. The Impact of Work of Hydraulic Compactor Type V8 from MTS on the Level of Vibrations Propagated to the Environment. Transportation Geotechnics and Geoecology, TGG 2017, 17-19 May 2017, Saint Petersburg, Russia. Procedia Eng. 2017, 189, 478-483. [CrossRef]

9. Czech, K.; Gosk, W. Impact of the Operation of a Tri-band Hydraulic Compactor on the Technical Condition of a Residential Building. Appl. Sci. 2019, 9, 336. [CrossRef]

10. Athanasopoulos, G.A.; Pelekis, P.C. Ground Vibrations from Sheet Pile Driving in Urban Environment: Measurements, Analysis and Effects on Buildings and Occupants. Soil Dyn. Earthq. Eng. 2000, 19, 371-387. [CrossRef]

11. Czech, K.; Gosk, W. Analysis of the Vibration Propagation Induced by Pulling out of Sheet Pile Wall in a Close Neighbourhood of Existing Buildings. Procedia Eng. 2016, 143, 1460-1467. [CrossRef]

12. Jongmans, D. Prediction of ground vibrations caused by pile driving: A new methodology. Eng. Geol. 1996, 42, 25-36. [CrossRef] 
13. Bata, M. Effects on Buildings of Vibrations Caused by Traffic. Build. Sci. 1972, 6, 221-246. [CrossRef]

14. Hao, H.; Ang, T.C.; Shen, J. Building Vibration to Traffic-induced Ground Motion. Build. Environ. 2001, 36, 321-336. [CrossRef]

15. Hunaidia, O.; Guana, W.; Nicks, J. Building Vibrations and Dynamic Pavement Loads Induced by Transit Buses. Soil Dyn. Earthq. Eng. 2000, 19, 435-453. [CrossRef]

16. Kouroussis, G.; Connolly, D.P.; Verlinden, O. Railway-Induced Ground Vibrations-A Review of Vehicle Effects. Int. J. Rail Transp. 2014, 2, 69-110. [CrossRef]

17. Bachmann, H. Vibration Problems in Structures. Practical Guidelines; Basel-Boston-Berlin, Birkhäuser: Basel, Switzerland, 1997.

18. Chyży, T.; Czech, K.R.; Malesza, M.; Miedziałowski, C. Investigations and Evaluation of Road Vibrations Influence in Historic Buildings Complex. Conserv. News J. Assoc. Monum. Conserv. 2009, 26, 499-509.

19. DIN 4150-3:1999. Structural Vibration. Part 3: Effects of Vibration on Structures; DIN Deutsches Institut für Normung: Berlin, Germany, 1999.

20. DIN 45669-2:2005. Measurement of Vibration Immission—Part 2: Measuring Method; DIN Deutsches Institut für Normung: Berlin, Germany, 2005.

21. BS 7385-1:1990. Evaluation and Measurement for Vibration in Buildings_Part 1: Guide for Measurement of Vibrations and Evaluation of their Effects on Buildings; British Standard Institution: London, UK, 1990.

22. BS 7385-2:1993. Evaluation and Measurement for Vibration in Buildings_Part 2: Guide to Damage Levels from Groundborne Vibration; British Standard Institution: London, UK, 1993.

23. BS 5228-4:1992. Code of Practice for Noise and Vibration Control Applicable to Piling Operations; British Standard Institution: London, UK, 1992.

24. BS ISO 4866:2010/ISO 4866. Mechanical Vibration and Shock-Vibration of Fixed Structures; Guidelines for the Measurement of Vibrations and Evaluation of their Effects on Structures; British Standard Institution: London, UK, 2010.

25. PN-B-02170:2016-12. Evaluation of the Harmfulness of Building Vibrations due to Ground Motion; PKN: Warsaw, Poland, 2016. (In Polish)

26. Lindner, S.; Pfeifer, G. Probleme der Schwinungsmessung an der Erdbodenoberfläche als Folge der Wellenausbreitung in den Obersten Erdbodenschichten (Problems in Vibration Measurements on Ground Surfaces due to Wave Propagation in the Top Soil Layer). In Proceedings of the Fortschritte der Akustik, 24. Jahrestagung für Akustik, DAGA 98, Zürich, Switzerland, 23-26 March 1998. (In German)

27. Kouroussis, G.; Mouzakis, H.P.; Vogiatzis, K.E. Structural Impact Response for Assessing Railway Vibration Induced on Buildings. Mech. Ind. 2017, 18, 803. [CrossRef]

28. Degan, G.A.; Coltrinari, G.; Lippiello, D. Analysis of Ground-Transducer Coupling in Monitoring Vibration from Railways: A Case Study. Int. J. Transp. Develop. Integr. 2017, 1, 290-300. [CrossRef]

29. Abratański, A.; Chełmecki, J.; Maciąg, E.; Tatara, T. Ground Vibration Transmission into Buildings in Cases of Municipal Transport Services. In Proceedings of the X Symposium "Seismic and paraseismic influences on buildings", Kraków, Poland, 27-28 November 2003; pp. 185-194. (In Polish)

30. Ulgen, D.; Ertugrul, O.L.; Ozkan, M.Y. Measurement of Ground Borne Vibrations for Foundation Design and Vibration Isolation of a High-Precision Instrument. Measurement 2016, 93, 385-396. [CrossRef]

31. Czech, K.; Gosk, W. Measurement of Surface Vibration Accelerations Propagated in the Environment; Transportation Geotechnics and Geoecology, TGG 2017, 17-19 May 2017, Saint Petersburg, Russia. Procedia Eng. 2017, 189, 45-50. [CrossRef]

32. Wang, F.; Yan, L.; Jiang, Q.; Tao, X. Measurement and Numerical Simulation of Ground and Subgrade Vibrations of Beijing Urban Rail Line 13. Adv. Civ. Eng. 2020. [CrossRef]

33. Hwang, J.H.; Tu, T.Y. Ground Vibration due to Dynamic Compaction. Soil Dyn. Earthq. Eng. 2006, 26, 337-346. [CrossRef]

34. Gao, G.; Chen, J.; Yang, J.; Meng, Y. Field Measurement and FE Prediction of Vibration Reduction Due to Pile-raft Foundation for High-tech Workshop. Soil Dyn. Earthq. Eng. 2017, 101, 264-268. [CrossRef]

35. Zhang, Y.; Zhang, N. Model for Predicting the Vibrations of Historical Timber Buildings due to Traffic Loads and its Experimental Validation. J. Low Freq. Noise Vib. Act. Control 2019, 38, 328-340. [CrossRef]

36. DIN EN 10056-1. Structural Steel Equal and Unequal Leg Angles-Part 1: Dimensions; DIN Deutsches Institut für Normung: Berlin, Germany, 1999. 
37. UNI 9916:2004. Criteria for the Measurement of Vibrations and the Assessment of their Effects on Buildings/Criteri di Misura e Valutazione Degli Effetti Delle Vibrazioni Sugli Edifice; UNI Ente Italiano di Normazione: Milano, Lombardia, Italy, 2004. (In Italian)

38. UNI 5783-66. Angular Beams with Equal Sides Round Edges/Travi Angolari a Lati Uguali Spigoli Tondi; UNI Ente Italiano di Normazione: Milano, Lombardia, Italy. (In Italian)

39. Masoumi, H.R.; Degrande, G.; Lombaert, G. Prediction of Free Field Vibrations Due to Pile Driving Using a Dynamic Soil-Structure Interaction Formulation. Soil Dyn. Earthq. Eng. 2007, 27, 126-143. [CrossRef]

40. Gosk, W.; Czech, K. A Stiffness Modulus and Poisson's Ratio of Soil Possibility Assessment on the Basis of Surface Vibration Acceleration Measurements. Acta Sci. Pol. Archit. 2018, 17, 15-23. (In Polish)

(C) 2020 by the author. Licensee MDPI, Basel, Switzerland. This article is an open access article distributed under the terms and conditions of the Creative Commons Attribution (CC BY) license (http://creativecommons.org/licenses/by/4.0/). 